\title{
Stable reverse bias or integrated bypass diode in HIP-MWT+ solar cells
}

\author{
Tadeo Schweigstill $^{1, *}$, Alma Spribille ${ }^{1}$, Jonas D. Huyeng ${ }^{1}$, Florian Clement ${ }^{1}$, and Stefan W. Glunz ${ }^{1,2}$ \\ ${ }^{1}$ Fraunhofer Institute for Solar Energy Systems ISE, 79110 Freiburg, Germany \\ 2 Department for Sustainable Systems Engineering, University Freiburg, 79098 Freiburg, Germany
}

Received: 30 June 2021 / Received in final form: 22 November 2021 / Accepted: 24 December 2021

\begin{abstract}
The Metal Wrap Through+ (HIP-MWT+) solar cell is based on the PERC concept but features two additional electrical contacts, namely the Schottky contact between $p$-type Si bulk and Ag $n$-contact and the metal-insulator-semiconductor (MIS) contact on the rear side of the cell below the $n$-contact pads. To prevent thermal hotspots under reverse bias, both contacts shall either restrict current flow or allow a homogenous current flow at low voltage. In this work we present both options. First the stable reverse bias characteristics up to $-15 \mathrm{~V}$ with a MIS contact using industrially manufactured SiON passivation and second, an integrated bypass diode using $\mathrm{AlO}_{X}$ as insulator in the passivation stack allowing current flows at approximately $V_{\text {rev }}=-3.5 \mathrm{~V}$ depending on the chosen screen-print paste. The examined Schottky contacts break down at around $V_{\text {rev }}=-2.5 \mathrm{~V}$. Reverse bias testing of the cells reveals a solid performance of the cells under reverse bias and an average conversion efficiency of $\eta=21.2 \%\left(\mathrm{AlO}_{X}\right)$ and $\eta=20.7 \%(\mathrm{SiON})$, respectively.
\end{abstract}

Keywords: MWT solar cells / integrated by-pass diode / silicon / passivation / PERC / back contact

\section{Introduction}

The passivated emitter and rear cell (PERC) [1] technology is now the standard technology in industrial production [2] and it can be combined with the metal wrapped through approach (MWT) [3] resulting in the MWT-PERC concept [4]. However, this cell concept required additional process steps in order to insulate the through-metallization from the Si bulk and thus complicates the manufacturing sequence.

A simplified version of the MWT-PERC concept is the High-Performance Metal Wrap Through $+($ HIP-MWT + ) cell where only one additional process step (drilling of vias) is required to create a PERC with $p$ - and $n$-contact located on the rear side of the cell [5]. This back-contact configuration results in several obvious benefits such as a rear side module interconnection and reduced front side shading.

However, wrapping the metal through the Si bulk and placing the external $n$-contact on the rear also introduces new electrical contacts, namely the Schottky contact between $p$-type Si bulk and $\mathrm{Ag} n$-contact in the vias and the metal-insulator-semiconductor (MIS) contact on the rear side of the cell below the $n$-contact pads. In order to clarify the cell structure, a schematic cross-section of the cell and the resulting electrical contacts are illustrated in Figure 1.

These additional contacts offer the following customization potential.

\footnotetext{
* e-mail: tadeo.schweigstill@ise.fraunhofer.de
}

- If the contacts are designed for electrical insulation, a cell with a stable reverse bias characteristic is created, comparable to a regular PERC. This means that reverse current flow under partial shading in the module is restrained and thus thermal hotspots [6] are prevented, allowing a standard module integration of the cells that could be certified by the International Electrotechnical Commission.

- However, one can also exploit the contacts by designing dielectric layers which insulate under forward bias but allow a current flow through the cell under reverse bias across a large area. This way an integrated bypass diode is built into the cell with only little additional production effort. In this case the term "integrated bypass" refers to a cell setup which allows a current flow under reverse bias through the cell across a sufficiently large area without harming the cell by the dissipated power. A similar feature has already been reported in the interdigitated back contacted (IBC) cell concept [7]. The implementation of the integrated bypass diode could lead to a significant simplification of the module assembly since no additional bypass diodes are required in the module and a single shaded cell is not leading to an outage of a whole 20-cellstring which could reduce the power output of a module by 33 percent.

In order to find suitable passivation stacks for the insulating and conducting properties in the MIS contacts, it seems appealing to compare different passivation principles. First, a chemical passivation using $\mathrm{SiO}_{2}$ appears 


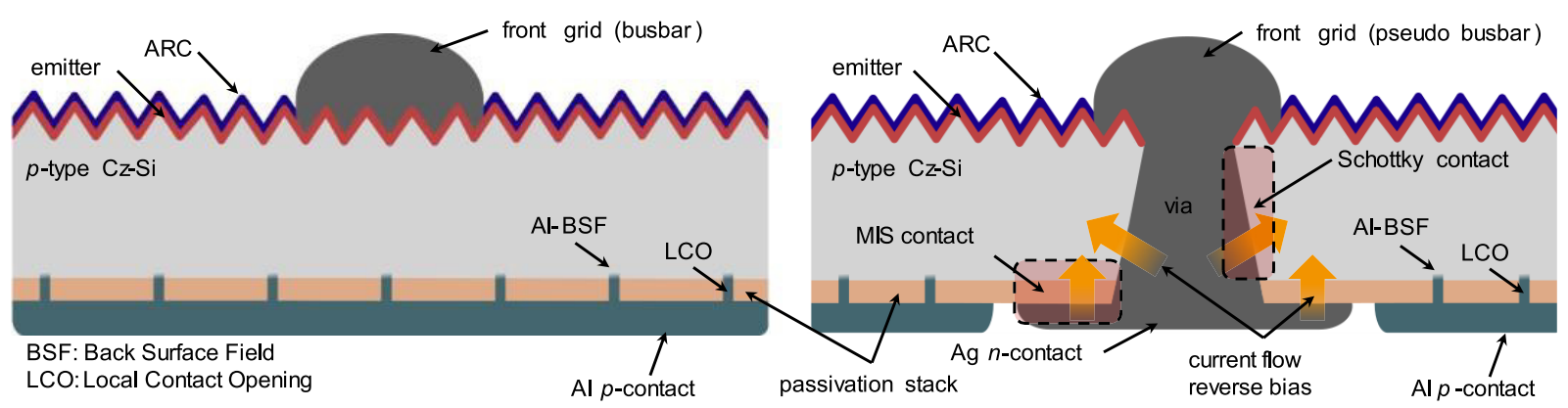

Fig. 1. Cross sections of PERC (left) and HIP-MWT + (right) cells showing the differences of the two cell concepts. The HIP-MWT + cell features the additional through-metallization (via) which creates two additional electrical contacts in the cell, namely the MIS contact and the Schottky contact. The vias allow locating the $n$-contact and $p$-contact next to each other on the rear side of the cell (right). The yellow arrows show the current flow under reverse bias for the $\mathrm{AlO}_{X}$ based passivation. This way the integrated bypass diode is realized.

suitable to create an isolating passivation stack. Second, a passivation based on the electrical field effect using $\mathrm{AlO}_{X}$ seems appropriate to function as a rectifying contact.

\section{Experimental}

For the experimental realization of different contacts, two solar cell precursor types are used. The precursors are sourced from different industrial partners, after the front and rear surface passivation has been applied. Both precursor types are made from $p$-type $\mathrm{Cz}-\mathrm{Si}$ and all passivation stacks are deposited using Plasma Enhanced Chemical Vapor Deposition (PECVD). The first stack is relying on the chemical passivation with a total thickness of $d \approx 160 \mathrm{~nm}$ consisting of a hydrogenated siliconoxynitride $(\mathrm{SiON})$ stack $(d \approx 75 \mathrm{~nm})$ capped with $\operatorname{SiN}_{X}(d \approx 85 \mathrm{~nm})[8]$. The second stack relying on the electrical field effect passivation with a total thickness of $d \approx 150 \mathrm{~nm}$ is consisting of an $\mathrm{AlO}_{X}$ stack $(d<20 \mathrm{~nm})$ and $\operatorname{SiN}_{X}$ capping $(d \approx 130 \mathrm{~nm})$ [9]. The metal side in the Schottky and MIS contact is created by a screen-printing process using a commercial non-fire-through paste. While formally referred to as $\mathrm{Ag}$, it is no pure metal due to the additives in the screen-printing paste. As the screenprinting paste contributes to the electric contacts, two non-fire-through screen-printing pastes with slightly different compositions were applied. More specifically, only paste 1 contains a small quantity of $5-10 \mathrm{wt} . \%$ of glass frit, while paste 2 is not containing any glass frit. For the frontside grid a commercial fire-through screenprinting paste was applied. The following manufacturing of test structures and solar cells was carried out using industrial production equipment located at the PV-TEC laboratory of Fraunhofer ISE [10].

Based on the described precursors, two types of dedicated test structures are manufactured: First a set of test structures to reveal the individual $I V$-characteristics of the MIS and Schottky contact, which show the reverse bias breakdown of the two contacts for different precursors and passivation stacks. Therefore, the different precursor types were metallized via screen printing. While the frontside (no anti-reflection coating) was fully metalized with an Al-paste to create a low resistance ohmic contact, the rear side was metallized with circular Ag-pads with diameters between $\phi=0.5 \mathrm{~mm}$ and $\phi=2.5 \mathrm{~mm}$. To create test samples with MIS as well as Schottky contacts, $50 \%$ of the test samples had the rear side passivation stack removed prior to metallization. After metallization, all samples were then contact fired at $T_{\text {peak }}=850{ }^{\circ} \mathrm{C}$. The samples were measured using an industrial 4-Point-Probe $I V$-setup. Two measurement pins were contacted on the front and two on the rear side, thus the $I V$-characteristics of the contacts were determined. The front side contact was proven to be ohmic, hence not influencing the measured rectifying behavior of Schottky and MIS contact. For the measurements, voltage sweeps from $-15 \mathrm{~V}$ to $+15 \mathrm{~V}$ were conducted with a step size of $10 \mathrm{mV}$, a delay of $50 \mathrm{~ms}$ and a current compliance of $1000 \mathrm{~mA}$.

Second, a set of test structures for lock-in thermography imaging (LIT) of the spatial breakdown was processed. These samples ran through the same process steps as the regular HIP-MWT + cells as shown in Figure 3. To further investigate the contact characteristics using LIT, the rear side metallization layout and the underlying local contact openings (LCO) were adapted. Instead of the regular rear side $n$-pads, a pad geometry variation was carried out with (a) circular pads reaching from $\phi=2 \mathrm{~mm}$ to $\phi=20 \mathrm{~mm}$ each with one via in the center of the pad and (b) elongated pads with varying length (width $=2 \mathrm{~mm}$ ) each with two vias. After screen printed metallization, the samples were contact fired at $T_{\text {peak }}=850{ }^{\circ} \mathrm{C}$ and measured in a commercial solar cell analysis system (LOANA, pv-tools [11]). Each sample was measured seven times with set voltages from $-1 \mathrm{~V}$ to $-10 \mathrm{~V}$, a LI-frequency of $18.75 \mathrm{~Hz}$ and a measurement duration of up to $300 \mathrm{~s}$ for low voltages.

Finally, HIP-MWT + cells with a 6 pseudo-busbar layout in M2 format were manufactured on the industrial precursors with the named industrial process tools at Fraunhofer ISE. The front and rear side of the final cells is illustrated in Figure 2. The HIP-MWT + cells were manufactured in five process steps in parallel to a PERC reference group as shown in Figure 3. In the first process step the precursors were processed in an industrial laser system (ILS500X, Innolas Systems [12]). Here, the HIPMWT + cells receive LCOs $(\phi=40 \mu \mathrm{m}$, pitch of $500 \mu \mathrm{m})$ 


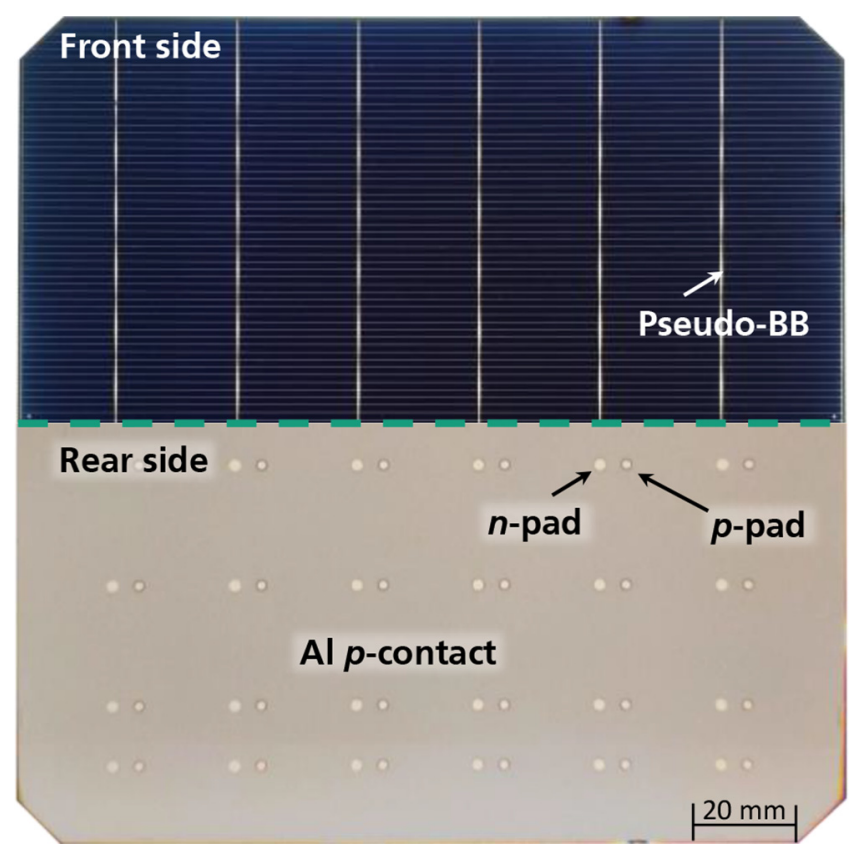

Fig. 2. Front and rear side of the manufactured HIP-MWT+ solar cell. Showing the front side metallization layout with 6 pseudo busbars on an M2 format $p$-type mono-crystalline silicon wafer.

and 48 vias $\left(\phi_{\text {rear }}=148 \mu \mathrm{m}, \phi_{\text {front }}=100 \mu \mathrm{m}\right)$ across the whole cell. In the following rear side metallization process, the via-filling is achieved by a controlled suction process of the vacuum chuck [13]. The following screen printing of the $\mathrm{Al}$ contact and front side grid were realized in parallel to standard PERC processes using an automated screen printer (XH2, ASYS TECTON GmbH). After contact firing the cell performance was measured in a commercial solar cell analysis system (customized, h.a.l.m. Elektronik $\mathrm{GmbH}$ [14]). Additionally, the manufactured cells were used for reverse bias cycling to demonstrate the long-term stability of the cells and the electrical contacts for 20 consecutive cycles at $-15 \mathrm{~V}$ (Forward bias at standard testing conditions for $100 \mathrm{~ms}$ and reverse bias for $40 \mathrm{~ms}$ with a compliance current of $I_{\text {rmax }}=-0.5 \mathrm{~A}$ ).

\section{Results and discussion}

The special features of the HIP-MWT + cells are the Schottky contact inside the via of the cell and the MIS contact on the rear side of the cell between the $n$-pad and the $p$-type silicon. To fully explore these contacts the two types of dedicated test structures were evaluated, and the results are presented in the following paragraphs. Finally, the findings were converted into fully functional solar cells and the performance of the contacts is demonstrated on cell level also determining the long-term stability of the contacts under reverse bias. The different experimental results were analyzed to provide extensive insight into the current potential of HIP-MWT + solar cells, based on current industrial fabrication sequences.

\subsection{Current-voltage characteristics of Schottky contact and MIS contact}

The isolating properties of the MIS contacts are investigated for the chemical SiON passivation stack, as shown in Figure 4 (left). The SiON passivation stack shows no breakdown under reverse bias up to $V_{\text {rev }}=-15 \mathrm{~V}$ independent from the chosen screen-print paste. This isolating property is sufficient for a regular module assembly with $20 \mathrm{Cz}$-Si solar cells in series in one module string $\left(20 \times V_{\mathrm{mpp}} \approx 11.6 \mathrm{~V}\right)$. The Schottky contact without the $\mathrm{SiON}$ passivation between the $\mathrm{Si}$ and $\mathrm{Ag}$ however, has a breakdown voltage between $V_{\text {rev }}=-2.5 \mathrm{~V}$ and $V_{\mathrm{rev}}=-3 \mathrm{~V}$ depending on the applied screen-print paste. In this case the Schottky barrier is overcome and a current is flowing under reverse bias. This current has the potential to create local thermal hotspots in the solar cell. Therefore, the effect and the consequences on cell level are illustrated by the LIT-images and further discussed in Section 3.2.

The Schottky contact on the $\mathrm{AlO}_{X}$ based precursor $\left(\mathrm{AlO}_{X}\right.$ removed) breaks at even lower voltages at approximately $V_{\mathrm{rev}}=-1.5 \mathrm{~V}$ to $V_{\mathrm{rev}}=-2.5 \mathrm{~V}$ again depending on the applied screen-printing paste. The differing breakdown voltages for the two Schottky contacts from the different precursors depend on the different bulk material of the precursors. Bulk resistivity measurement show, the precursor using the $\mathrm{AlO}_{X}$ based stack has a $50 \%$ lower base resistivity $(0.94 \pm 0.03 \Omega \mathrm{cm})$ than the precursor using the SiON based stack $(1.97 \pm 0.14 \Omega \mathrm{cm})$, which causes the lower contact resistance for the Schottky contact of the $\mathrm{AlO}_{X}$ based precursor.

The MIS contact using the $\mathrm{AlO}_{X}$ passivation stack shows a breakdown at approximately $V_{\text {rev }}=-3.5 \mathrm{~V}$ depending on the applied screen-print paste as shown in Figure 4 (right). This breakdown behavior of the $\mathrm{AlO}_{X}$ based MIS contact is similar to the breakdown of the Schottky contacts, which is needed for a controlled breakdown across a large area that would be required for a HIP-MWT + cell with an integrated bypass diode. If the MIS and Schottky contact are adapted to break in a small voltage window, the reverse current through the cell is distributed more evenly so that single spots with high currents are avoided. To express it differently, by substituting Ohm's law into the generalized power equation, we get:

$P=I^{2} R$

with power dissipated $P$, resistance $R$ and current $I$.

For the HIP-MWT + cell under reverse bias it means that if the local current $(I)$ is spread across a larger area and thus locally reduced, the locally dissipated power is also reduced, and thermal hotspots are prevented.

We suggest that the different additives in the paste significantly contribute to the reverse bias breakdown of the electrical contacts. Therefore, the type and concentration of paste additives seem suitable to further manipulate and developed the desired breakdown voltage of the Schottky and MIS contact. The crucial paste ingredient seems to be the glass frit which is only found in paste 1 


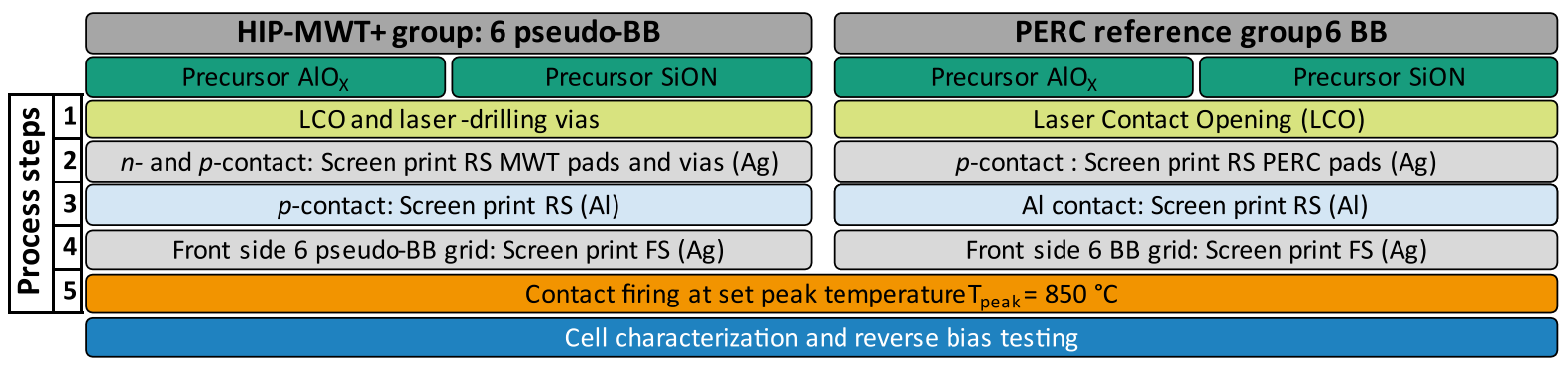

Fig. 3. Process steps for HIP-MWT + cells and PERC reference cells. The process steps for HIP-MWT + cells (left) are also reflecting the process steps for the test structures use for the LIT characterization. Merely the LCO and rear side metallization layouts are adapted.
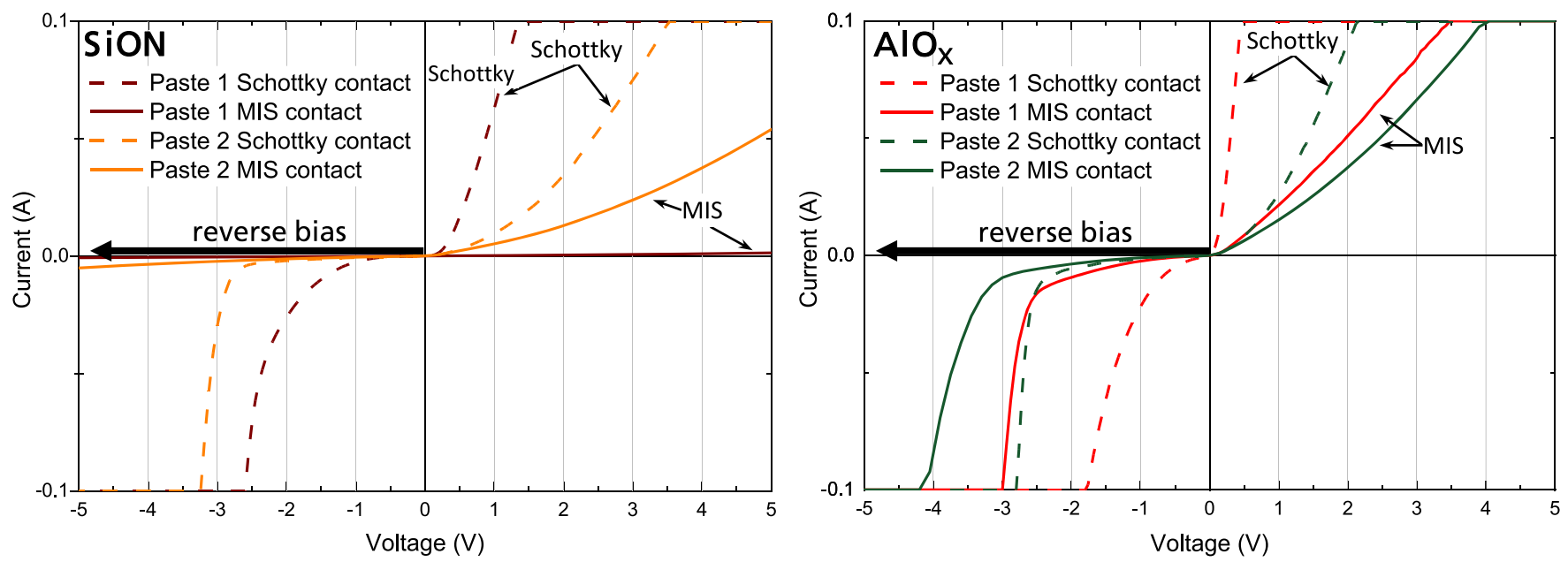

Fig. 4. Current voltage characteristics of quantitative test structures. For each contact one representative $I V$ characteristic is shown. The measured circular pads have a printed total area of $A=3.14 \mathrm{~mm}^{2}$, the actual contact area of $\mathrm{Ag}$ and $\mathrm{Si}$ is differing due to additions in the screen-print paste. The definition of the polarity is congruent with the regular operation of a solar cell for $V>0$ (forward bias) the $p$-contact is connected to the positive terminal of the measurement device. Therefore, in the 1st quadrant the non-ideal ohmic behavior of the electrical contact under forward bias is shown. While in the 3rd quadrant the rectifying contact behavior of the diode under reverse bias can be seen.

causing a more inhomogeneous contact area due to the glass frit penetrating the bulk and thus showing less abrupt $I V$-characteristics.

\subsection{Spatially resolved current flows of Schottky contact and MIS contact}

The $I V$-characteristics of the Schottky and MIS contact are valuable to evaluate the breakdown of the individual contacts. However, in the HIP-MWT + the contacts are located in direct vicinity and thus inevitably interact with one another. To illustrate the occurrence around the vias and below the $n$-pads, LIT imaging was used as an informative characterization method.

First, the LIT images qualitatively confirm the findings of the $I V$-characteristics (Fig. 5). It appears that the MIS contact using $\mathrm{SiON}$ as insulator did not emit heat. Fortunately, the Schottky contacts on the same sample also show only minor heat emission. Most of the LIT signals are counted near the sample edges at the passivation defects which are congruent with the holding pins during PECVD as shown in Figure 5 (left). Since the emitted heat/power is resulting from local reverse currents it can be concluded that no currents flow through the MIS contact, only very small currents flow through the interface of viametallization and Si bulk and the major shunts at the wafer edges are unrelated to the HIP-MWT + concept. These major shunts shown in the LIT image, could lead to hotspots in PERC solar cells if no bypass diodes are applied in the module. Furthermore, the reverse currents of the test sample in M2 format with 24 vias are adequately low with only $I_{\mathrm{rev}}=-136 \mathrm{~mA}$ at $V_{\mathrm{rev}}=-10 \mathrm{~V}$. Therefore, the Schottky contacts in the sample do not have a negative impact in reverse bias and can be used in solar cells with a stable reverse bias.

The test sample using the $\mathrm{AlO}_{X}$ passivation shows a different performance, as expected from the different $I V$ characteristics of the contacts. At small reverse voltages $V_{\text {rev }}=-1 \mathrm{~V}$ no heat emission is detected by the infrared camera of the measurement setup. With increasing 

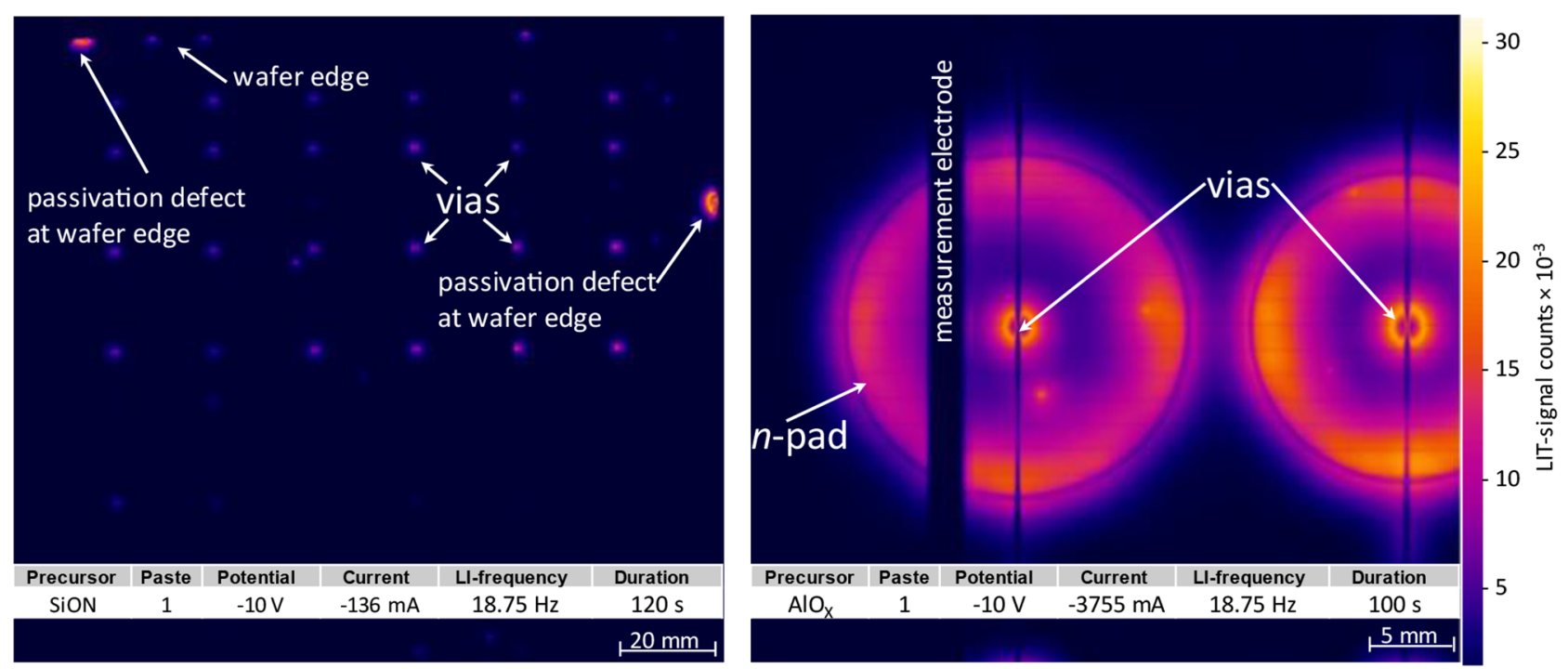

Fig. 5. LIT images full sample with $\mathrm{SiON}$ passivation stack (left) and zoom on Schottky and MIS contact $\left(\mathrm{AlO}_{X}\right)$ (right). The test sample with the SiON passivation stack (left) shows a significantly lower reverse current and heat emission restricted to the 24 vias and the passivation defect at the wafer edge. The MIS contact $\left(\mathrm{AlO}_{X}\right)$ of the largest $n$-pads $(\phi=20 \mathrm{~mm})$ of the test structures (right) illustrating the increased current flow at the pad edges. Also indicated in the table below the pictures is the significantly higher current for the whole sample.

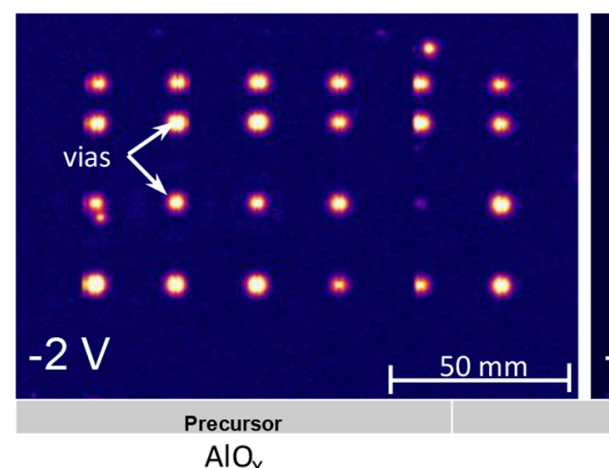

$\mathrm{AlO}_{\mathrm{x}}$

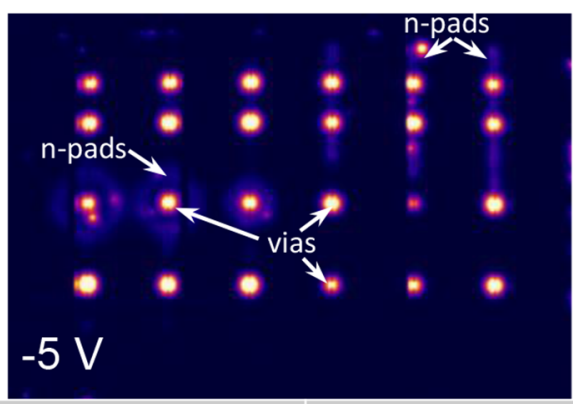

Paste

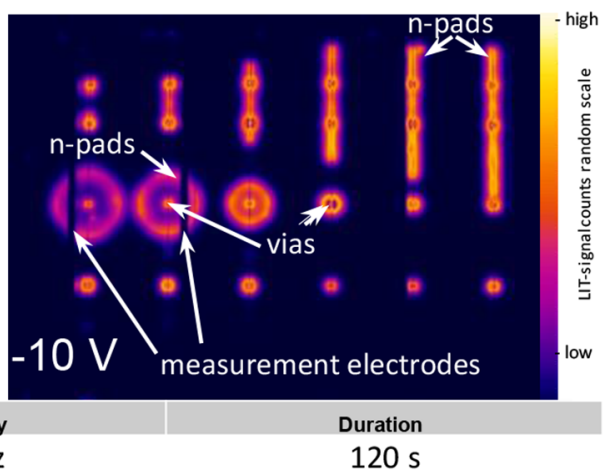

$120 \mathrm{~s}$

Fig. 6. LIT images of Schottky and MIS contact $\left(\mathrm{AlO}_{X}\right)$ combined in via and $n$-pads. At $-2 \mathrm{~V}$ the current passes through the Schottky contact in the vias. At $-5 \mathrm{~V}$ the current passes through the Schottky contact in the vias as well as through the MIS contact below the $n$-pads. At $-10 \mathrm{~V}$ all MIS contacts contribute significantly to the current flow. Note that the LIT-signal count scales of the pictures are unrelated to visualize the low counts at low voltages.

potential, a small current flows and the resulting heat emission is registered at the matrix of the 24 vias of the test sample as shown in Figure 6, confirming a small current flow of $I_{\mathrm{rev}}=-200 \mathrm{~mA}\left(V_{\mathrm{rev}}=-2 \mathrm{~V}\right)$ in the via-areas. At higher voltages such as $V_{\text {rev }}=-5 \mathrm{~V}$ (Fig. 6, middle), additional heat signals are detected at the MIS contacts below the circular and elongated $n$-pads clearly confirming a current flow through the $\mathrm{AlO}_{X}$ passivation stack in the designated areas. At $V_{\text {rev }}=-10 \mathrm{~V}$ (Fig. 6 , right), all $n$-pads are emitting significant heat revealing the sample's $n$-pad layout. The current over the whole sample sums up to $I_{\mathrm{rev}}=-3755 \mathrm{~mA}$. Here it becomes obvious that more heat is emitted at the edges of the large $n$-pads as well as from the elongated pads with a higher edge to area ratio, leading to the conclusion that higher currents are present in the MIS contact near the $n$-pad edges. This effect becomes clearer when zooming in on the circular pads as shown in Figure 5 (right).

In additional experiments, the oddments of the test samples $\left(\mathrm{AlO}_{X}\right)$ were used for $I V$-measurements. The largest $n$-pad was diced into to $5 \times 5 \mathrm{~mm}$ large samples and $I V$-measured from front to back (no emitter in this sample). The sample pieces near the pad edge showed significantly higher currents at the same voltage than the center pieces. Thus, confirming the increased current flow at the pad edges. These findings and the 3D-images of the measured pads suggest a correlation between increased pad height and increased current flow as shown in Figure 7. We suggest that this correlation originates from the locally increased applied quantity of the paste and the associated 

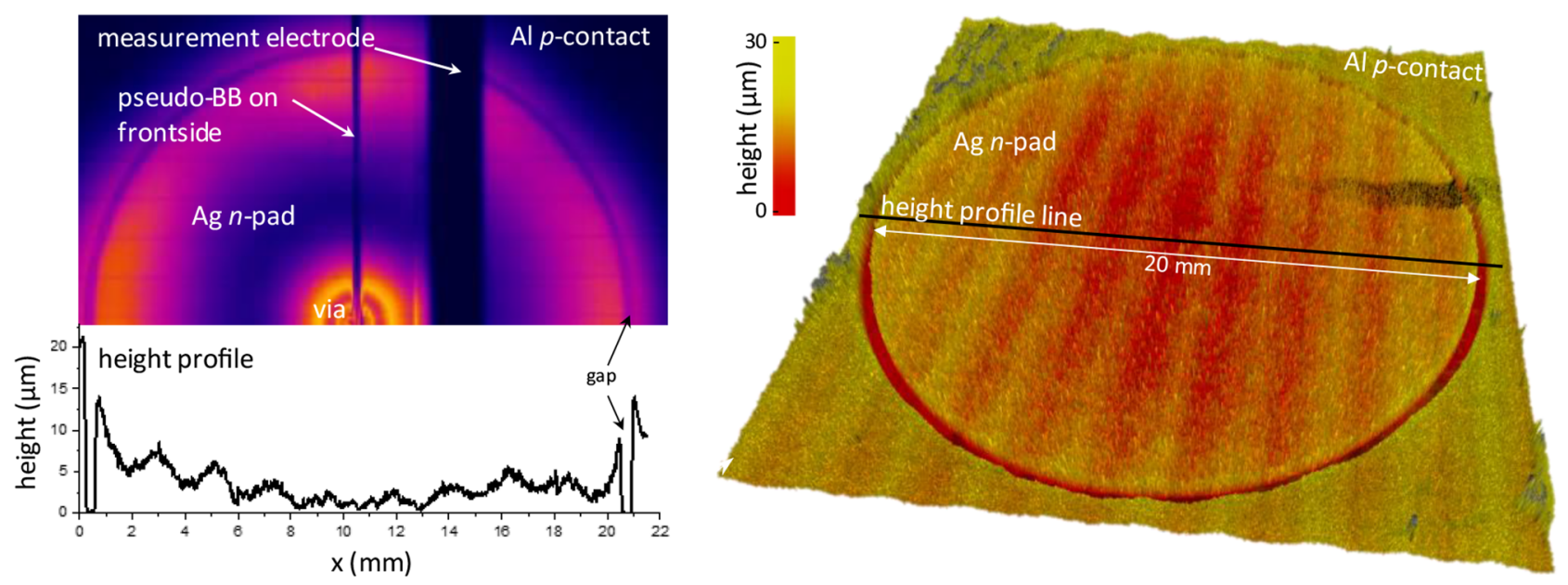

Fig. 7. Pad height in correlation to increased reverse current flow illustrated in LIT measurement. The locally increased current flow at the n-pad edges (left) in relation to the pad's height profile (below). In addition, a 3D-image of the $n$-pad showing an increase pad height at the circular edges (right)where reverse currents are high. Note that the frequent linear embossments in the 3D-image are measurement artefacts originating from the stitching of the microscopic images.

Table 1. Selected cell parameter results at the 1st and 20th measurement cycle of HIP-MWT + with different passivation stacks. The relative changes between 1st and 20th measurement are indicated in brackets.

\begin{tabular}{llllll}
\hline Rear passivation & Efficiency $(\%)$ & $V_{\mathrm{OC}}(\mathrm{mV})$ & $\mathrm{j}_{\mathrm{sc}}\left(\mathrm{mA} / \mathrm{cm}^{2}\right)$ & $\mathrm{FF}(\%)$ & $\mathrm{j}_{\mathrm{rev}}\left(\mathrm{mA} / \mathrm{cm}^{2}\right)$ \\
\hline $\mathbf{A l O}_{\boldsymbol{X}}$ & 21.2 & 671.4 & 40.2 & 78.65 & 9.7 \\
AlO $_{\boldsymbol{X}}$ after 20 cycles & $21.0(-0.9 \%)$ & $671.9(+0.1 \%)$ & $40.1(+0.2 \%)$ & $77.64(-1.3 \%)$ & $17.4(+44 \%)$ \\
SiON & 20.7 & 665.5 & 39.6 & 78.71 & 1.4 \\
SiON after 20 cycles & $20.7(0.0 \%)$ & $666.2(-0.1 \%)$ & $39.6(0.0 \%)$ & $78.76(+0.1 \%)$ & $2.4(+42 \%)$ \\
\hline
\end{tabular}

increased amount of paste additives which lead to a lower contact resistance in the MIS contact. This finding could be used to manipulate the MIS contact when a reverse bias breakdown of the MIS contact is intended. However, further investigation is needed to fully comprehend the correlation and the altered breakdown.

\subsection{Cell results and long-term stability of Schottky and MIS contacts}

The findings from both sets of test structures are complemented by the solar cell performance results. For each group, 20 cells were measured, and the average cell parameter results are shown in Table 1 . The higher initial performance of the precursor using the $\mathrm{AlO}_{X}$ passivation stack can be explained by an overall superior quality of the precursor and is not necessarily related to the different passivation mechanisms, since very low surface recombination rates for $\mathrm{SiO}_{X}$ were demonstrated [15]. However, it becomes clear that the Schottky contact as well as both MIS contacts using the $\mathrm{AlO}_{X}$ and $\mathrm{SiON}$ as insulators are functional and lead to reasonable forward bias performances in the manufactured cells with average conversion efficiencies of $\eta=21.2 \%$ for the $\mathrm{AlO}_{X}$ passivated samples and $\eta=20.7 \%$ for the SiON passivated samples under STC, respectively.
In terms of the long-term stability of the solar cells, the effect of repeated reverse bias on the Schottky and MIS contact are highly relevant. Therefore, the performance of the solar cells is observed over 20 alternating cycles with forward and reverse bias. The final cell results after testing in contrast to the initial cell results are given in Table 1 and the performance shift over the 20 measurements for one SiON and one $\mathrm{AlO}_{X}$ passivated cell is plotted and compared to each one reference PERC in Figure 8.

The cell parameter results over the 20 measurements show a consistent performance for the two PERC reference cells with the $\mathrm{AlO}_{X}(\mathrm{PERC} \mathrm{A})$ and $\mathrm{SiON}$ passivation stacks (PERC S). The HIP-MWT + using the SiON passivation (MWT S) performs similarly with a neglectable parallel resistance $\left(R_{p}\right)$ decrease after the fourth measurement cycle. The only significant performance loss is observed for the HIP-MWT + using the $\mathrm{AlO}_{X}$ passivation (PERC A). The performance deterioration can be assigned to an increase in the reverse current-density $\left(\mathrm{j}_{\mathrm{rev}}\right)$ which goes hand in hand with the deterioration of $R_{p}$, leading to a reduced conversion efficiency.

The most significant deterioration is observed after the first bias. Afterwards all cell parameters are more stable, and the cell deterioration saturates quickly. We suggest that the increase in $\mathrm{j}_{\mathrm{rev}}$ is caused by percolation bias [16]. Hence, the repeated reverse bias creates conducting 

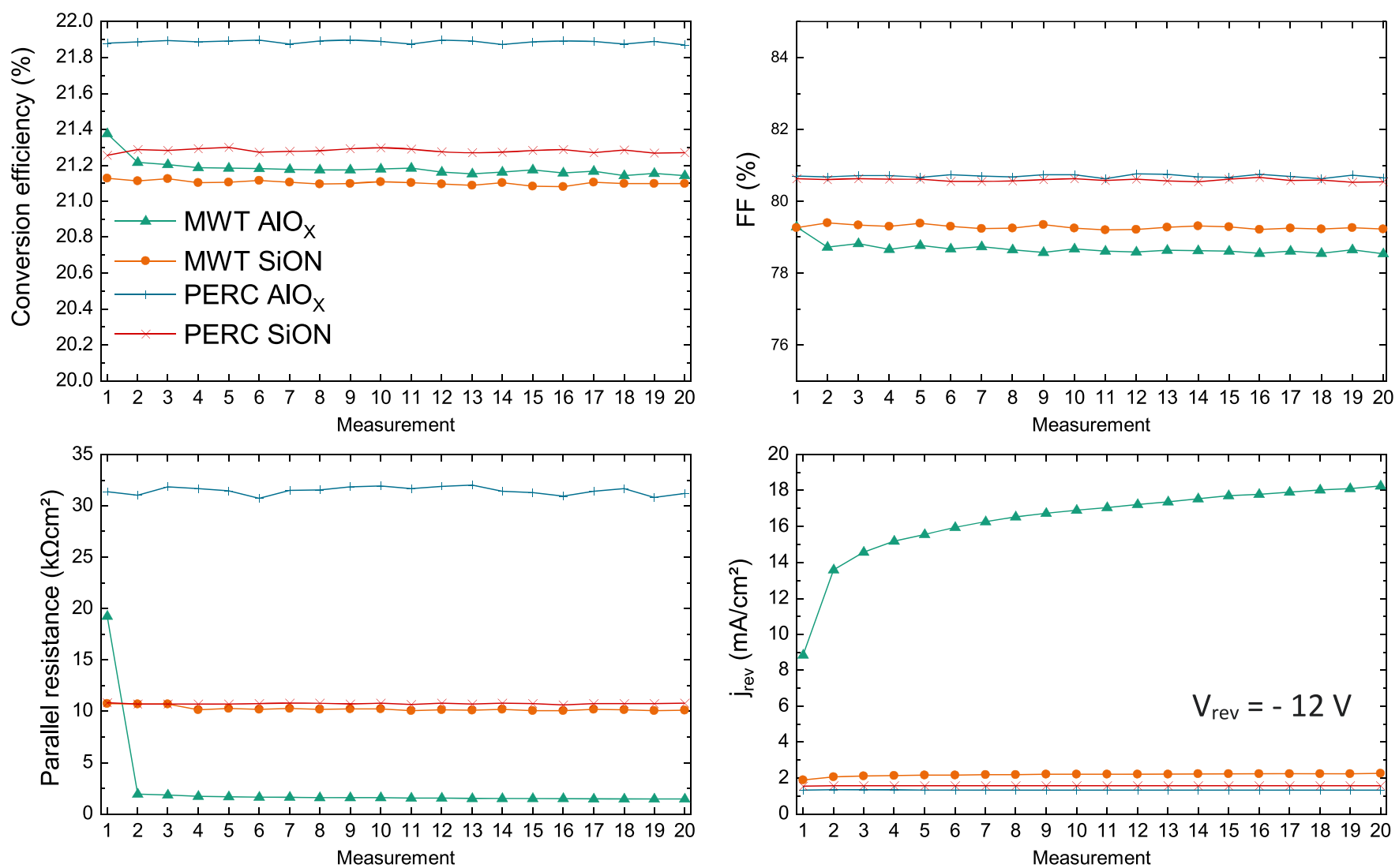

Fig. 8. Cell parameter shift over 20 consecutive reverse bias cycles. With the efficiency $\eta$, the fill factor FF, the parallel resistance $R_{p}$ and the reverse current-density $\mathrm{j}_{\mathrm{rev}}$ at a reverse bias of $V_{\mathrm{rev}}=-12 \mathrm{~V}$. The HIP-MWT + cell that uses the $\mathrm{AlO}_{X}$ is significantly affected by the reverse bias. The increase in reverse current-density goes hand in hand with the deterioration of $R_{p}$ which has the negative impact on the FF.

pathways for charge carriers in the passivation stack which systematically decreases the resistance of Schottky and MIS contact. The first pathways for the current may depend on the inhomogeneous contact surface due to the additives in the paste. Thus, this effect could even be reduced by choosing a screen-printing paste specifically optimized towards this function.

Nevertheless, the cells are stabilizing over the 20 cycles in this first proof of concept. The deterioration in a justifiable range of only $\Delta \eta_{\text {abs }} \approx 0.2 \%$ is manageable when considering the potential of an integrated by-pass diode in the HIP-MWT + cells.

\section{Conclusion}

In this work, we report on a fabrication process of two functional configurations of industrially manufactured HIP-MWT + solar cells. One with a stable reverse bias characteristic using $\mathrm{SiON}$ as passivation and another with an integrated bypass diode using $\mathrm{AlO}_{X}$ as passivation. The two relevant electrical contacts in the HIP-MWT + cells are the Schottky and the MIS contact.
Dedicated test structures show the specific behavior under reverse bias and reveal the individual as well as the combined breakdown behavior of these contacts. While the MIS contact using SION as insulator shows no breakdown under reverse bias up to $-15 \mathrm{~V}$, the breakdown of the MIS contact using $\mathrm{AlO}_{X}$ as insulator breaks at a reverse bias of approximately $V_{\text {rev }}=-3.5 \mathrm{~V}$. This breakdown voltage can be actively manipulated by the applied screen-print paste (depending on additives) and the pad-geometry. The Schottky contact breaks at voltages between $V_{\text {rev }}=-1.5 \mathrm{~V}$ to $V_{\text {rev }}=-3 \mathrm{~V}$ strongly depending on the precursor and again screen-print paste. However, the breaking of the Schottky contact in the cells using the SiON passivation is not problematic since only minor heat is emitted at very low reverse bias currents of $\mathrm{j}_{\mathrm{rev}}=-2.4 \mathrm{~mA} / \mathrm{cm}^{2}$ (M2 wafer 48 vias, $V_{\text {rev }}=-12 \mathrm{~V}$ after 20 test cycles). The Schottky contact in the cells using $\mathrm{AlO}_{X}$ as passivation allow higher currents which supports the overall current flow under reverse bias for this cell configuration. The cumulated reverse bias currents through Schottky and MIS contact are $\mathrm{j}_{\mathrm{rev}}=-17.4 \mathrm{~mA} / \mathrm{cm}^{2}$ (M2 wafer 48 vias, $V_{\text {rev }}=-12 \mathrm{~V}$ after 20 test cycles). 
The final cell results show that both cell configurations give functional cells. While the SiON passivation shows a good long-term stability in first tests, a slight performance degradation $\left(\Delta \eta_{\mathrm{abs}}=-0.2 \%\right)$ can be seen in the $\mathrm{AlO}_{X}$ passivation functioning as the by-pass diode. However, the effect of deterioration lessens over time while remaining in an acceptable range.

The authors would like to acknowledge the laboratory work and general help of their coworkers at Fraunhofer Institute for Solar Energy Systems ISE, especially the colleagues working in the PVTEC BE laboratory that supported the manufacturing and characterization of the test samples and final cells.

\section{Author contribution statement}

T. S. executed the experiments, the characterization, and the interpretation of the resulting data. He also wrote the manuscript with input from all authors. A. S. and J. H. initiated and assisted with the general design of the experiments and supported with the interpretation of the results. F.C. and S.G. directed this research study and focused the resources and attention onto particular areas of interest.

\section{References}

1. A.W. Blakers, A. Wang, A.M. Milne, Appl. Phys. Lett. 55, 13 (1989)

2. ITRPV, International Technology Roadmap for Photovoltaic (ITRPV) - Results 2018, 2019
3. E. Van Kerschaver, S. De Wolf, J. Szlufcik, Conference Record of the Twenty-Eighth IEEE photovoltaic Specialists Conference (2000), pp. 209-212

4. B. Thaidigsmann, J. Greulich, E. Lohmüller, S. Schmeißer, F. Clement, A. Wolf, D. Biro, R. Preu, Sol. Energy Mater. Sol. Cells 106, 89 (2012)

5. B. Thaidigsmann, Ph.D. thesis, University of Tübingen, 2013

6. S. Michael, E.L. Meyer, Sol. Energy Mater. Sol. Cells 94, 106 (2010)

7. H. Chu, A. Halm, V.D. Mihailetchi, G. Galbiati, R. Kopecek, Energy Procedia 92, 540 (2016)

8. C. Schwab, M. Hofmann, R. Heller, J. Seiffe, J. Rentsch, R. Preu, Phys. Stat. Solidi (a) 210, 11 (2013)

9. P. Saint-Cast, D. Kania, M. Hofmann, J. Benick, J. Rentsch, R. Preu, Appl. Phys. Lett. 95, 15 (2009)

10. Fraunhofer ISE, Neue Technologien und Solarzellenwirkungsgrade aus dem Fraunhofer ISE PV-TEC press release 26, 2019

11. pv-tools GmbH, [Online]. Available: http://www.pv-tools. de/products/loana-system/loana-start.html. [Accessed 17 06 2021]

12. InnoLas Solutions, [online]. Available: https://www.innolassolutions.com/laser-machines-products/linexo-linear-tablelaser-machine/. [Accessed 1706 2021]

13. E. Lohmüller, B. Thaidigsmann, S. Werner, F. Clement, A. Wolf, D. Biro, R. Preu, in Proceedings of the 27th European Photovoltaic Solar Energy Conference and Exhibition, 2AO.2.6 (2012)

14. h.a.l.m. Elektronik GmbH, [online]. http://www.halm.de/en/ products/production/solar-cell.html [Accessed 1706 2021]

15. S. Duttagupta, F.J. Ma, B. Hoex, A.G. Aberle, IEEE 39th Photovoltaic Specialists Conference (PVSC), (2013)

16. Z. Gingl, C. Pennetta, L.B. Kiss, L. Reggiani, Semicond. Sci. Technol. 11, 12 (1996)

Cite this article as: Tadeo Schweigstill, Alma Spribille, Jonas D. Huyeng, Florian Clement, Stefan W. Glunz, Stable reverse bias or integrated bypass diode in HIP-MWT + solar cells, EPJ Photovoltaics 13, 5 (2022) 